\title{
Progressive Camptocormia and Dropped Head Syndrome Induced By Chemoradiotherapy for Base of Tongue Carcinoma. Case Report
}

Allan Hiroshi de Araujo Ono ${ }^{1}$, Flavio Gerardo Benites Zelada1, Douglas Kenji Narazaki ${ }^{1 *}$, Willian Gemio Jacobsen Teixeira ${ }^{1}$, Raphael Martus Marcon ${ }^{1}$, Alexandre Fogaça Cristante ${ }^{2}$ and Tarcísio Eloy Pessoa Barros Filho²

${ }^{1}$ Spine Surgery Division, Institute of Orthopedics and Traumatology, Hospital of the University of Medical School Clinics, Brazil

${ }^{2}$ Spine Surgery Division, Laboratory of Medical Investigation, Institute of Orthopedics and Traumatology, Hospital of the University of Medical School Clinics, Brazil

\begin{abstract}
Background: Camptocormia (bent spine syndrome) is an acquired postural disease characterized by forward flexion of the thoracolumbar spine. Dropped head syndrome is characterized by severe weakness of the cervical paraspinal muscles, resulting in gradual sagging of the head and culminating in the classic chin-on-chest deformity.

Objective: To report, for the first time, a case of dropped head syndrome and camptocormia in the same patient.

Methods and results: A 68-years-old man was diagnosed with base tongue squamous cell carcinoma, surgically removed. He had local recurrence and underwent radiosensitive chemotherapy and radiotherapy (35 fractions of 70Gy). After 12 months, he developed progressive cervical extensor muscle weakness, and a flexible chin-on-chest deformity, treated with passive reduction and C3-T3 fixation. Three months later, camptocormia was diagnosed, and initially treated conservatively. Ten months later, the deformity became rigid, and he was operated with Ponte and pedicle subtraction osteotomies in thoracic and lumbar spine, and $\mathrm{C} 3$ to sacrum arthrodesis with instrumentation. The patient resumed professional activities by the third month. Oncological screening showed no tumoral recurrence or distant metastases, after one year from last surgery.
\end{abstract}

Conclusion: This is the first report of dropped head syndrome and camptocormia in the same patient, due radiosensibilizing chemotherapy for a nasopharyngeal carcinoma.

Keywords: Spine; Camptocormia; Radiotherapy; Lymphatic irradiation; Tongue neoplasms; Cervical vertebrae

\section{Introduction}

\section{Camptocormia}

Camptocormia (bent spine syndrome) is an acquired postural disease characterized by forward flexion of the thoracolumbar spine. Camptocormia leads to lumbar kyphosis and increases during walking or standing and completely disappears in supine position [1]. The majority of cases of camptocormia of muscular origin are related to a primary idiopathic axial progressive myopathy of late onset, in elderly patients. Causes of secondary camptocormia are numerous. They must be carefully assessed and ruled out before considering the diagnosis of primary axial myopathy [2].

Camptocormia is frequently associated with Parkinson's disease. Other causes include dystonia, multisystem atrophy, Alzheimer's disease, myopathies, motor neuron diseases, myasthenia, chronic inflammatory demyelinating polyneuropathy, Amyotrophic Lateral Sclerosis (ALS). Rare causes include adverse reactions of drugs, disc herniation, arthritis, spinal trauma, paraneoplastic disorders, or psychiatric disease (more rarely than previously thought). Camptocormia can also be idiopathic [1,3]. Psimaras et al. reported the case of a patient who developed typical camptocormia years after having received extended-field radiotherapy. The authors made a literature review with other 32 cases with radiotherapy as the probable cause [3].

Camptocormia is diagnosed upon clinical investigations, imaging of the brain or spine, electromyography or muscle biopsy [1]. Management of camptocormia is still a challenge and the literature on this topic is limited. There is no specific pharmacologic treatment for primary axial myopathy [2]. Treatment is limited on supportive conservative measures, withdrawal of causative drugs, electroconvulsive therapy, surgical correction or deep brain stimulation and these are effective only in few patients [1]. Conservative management should include appropriate drug therapy for the medically treatable conditions. Physical therapy focusing on strengthening of the paraspinal muscles can be considered. Braces can also be indicated with limited use, as they can worse the deformity with the long-term use, decreasing muscular strength. General activities, walking with a cane, physiotherapy, and exercises should be encouraged [2].

Despite conservative measurements, the disease may progress and cause a debilitating deformity. The flexion of the spine may lead to sagittal imbalance and ultimately to devastating condition, such as the dropped head syndrome and the chin-on-chest deformity. The positive sagittal imbalance results in a anterior translation of the head compared to the normal center of gravity: for every inch of translation, there is an increase in 10 pounds in the weight of the head, and this could be an etiology for the dropped head syndrome $[4,5]$.

Wadia et al. [6] reported two cases of camptocormia in patients with Parkinson's disease treated with corrective spinal surgery. Despite prolonged postoperative courses, including complications and the need for multiple revisions, both patients benefited from the procedures [6]. In a literature review in cases involving Parkinson's disease and camptocormia, the results showed that there is high rate of complications when spinal surgery is performed. The authors

*Corresponding author: Douglas Kenji Narazaki, Spinal Tumors Division, Institute of Orthopedics and Traumatology, Hospital of the University of Medical School Clinics, Brazil, Tel: (+55 11) 2661-6912; E-mail: douglasnarazaki@gmail.com

Received September 01, 2015; Accepted October 04, 2015; Published October 11, 2015

Citation: Ono AHA, Zelada FGB, Narazaki DK, Teixeira WGJ, Marcon RM, et al. (2015) Progressive Camptocormia and Dropped Head Syndrome Induced By Chemoradiotherapy for Base of Tongue Carcinoma. Case Report. J Clin Case Rep 5: 613. doi:10.4172/2165-7920.1000613

Copyright: (C) 2015 Shkalim-Zemer V, et al. This is an open-access article distributed under the terms of the Creative Commons Attribution License, which permits unrestricted use, distribution, and reproduction in any medium, provided the original author and source are credited. 
created and algorithm for treatment choice indicating spinal surgery only to patients with coexisting spinal stenosis causing radiculopathy or myelopathy. Patients with Parkinson's and camptocormia without spinal stenosis may be considered candidates for deep brain stimulation, but the results are confusing [7]. Therefore, surgery should be reserved for patients who have a significant deformity and can tolerate a surgical procedure. Proper counseling must be offered to the patient following treatment. Still, recent studies by Smith et al. [8] showed that correction of the spine deformity lead to great improvements in quality of life, even with the increased risk for complications from surgery, in the elderly population.

\section{Dropped head syndrome and chin-on-chest deformity}

Dropped head syndrome is characterized by severe weakness of the cervical paraspinal muscles, resulting in gradual sagging of the head and culminating in the classic chin-on-chest deformity. In contrast with other cervical kyphotic deformities, such as ankylosing spondylitis, dropped head syndrome is often correctable with passive neck extension [9]. There are two proposed etiologies for the development of dropped head syndrome [10]. One theory suggests that excessive anterior cervical muscle tension precipitates the syndrome, as in diseases such as multiple system atrophy [11]. An alternative theory postulates that the dropped head syndrome results from weakness or atrophy of the posterior cervical muscles. The most favored hypothesis for the etiology of the dropped head syndrome proposes that the cause is an injury to, and fatigue of the paraspinal musculature with secondary kyphotic postural changes and age-dependent loss of tissue elasticity [4]. Petheram et al. [12] proposed that an increase in the kyphosis of the thoracic vertebrae, coupled with the weight of the head, results in an anterior shift of the center of gravity. As the head becomes progressively more flexed and translates more anteriorly, the lever arm that acts on the cervical spine stresses the neck extensors. With time, the neck extensors become overstretched and can no longer generate enough tension to raise or extend the head when the patient is upright.

\section{Radiotherapy and spine deformity}

Johansson et al. [13] described cervical pain in 18 patients who received mantle field radiation therapy for Hodgkin's disease. Late neurological effects of radiotherapy for Hodgkin's disease included cervical and lumbar plexopathy, myelopathy and weakness of paraspinal muscles [14]. Gosh et al. [15] retrospectively reviewed 251 cases of neuromuscular complications of radiotherapy treatment of adult cancer at Mayo Clinic, and identified 21 myopathy cases, 13 due to Hodgkin's lymphoma, 2 that were due to tongue carcinoma e 1 in a nasopharyngeal carcinoma patient. Of these patients, $43 \%$ presented clinically with the dropped head syndrome. The mean latency between radiation exposure and the onset of symptoms was 15 years (ranging from 2 to 45 years) [15]. It is suggested that lower motor neuron and peripheral nerves can be injured by high-dose radiation [16]. Others believe that nerves could suffer ischemic injuries that could disturb conduction proprieties of axons but must of cases presented in literature corroborates with the neurogenic cause [17]. The case reported here, as we know, is the first describing progressive cervical and thoracic deformity, both conditions associated with high-dose radiotherapy and radiosensitive chemotherapy for carcinoma of the base of tongue, with dropped head syndrome and camptocormia surgically treated.

\section{Case Report}

The patient is a 68-years-old man, diagnosed in 2009 with a squamous cell carcinoma of base of tongue, surgically removed in
September 2010. By May 2011, local recurrence was diagnosed, and the patient was submitted to radiosensitive chemotherapy, followed by radical radiotherapy, with 35 fractions of 70 Gy. By July 2012, our spine oncology group evaluation was requested, because the patient was referring difficulty in extending the head and also difficulty in swallowing, and a progressive cervical deformity was observed in the previous few months. At that appointment, he presented paraspinal cervical weakness, chin-over-chest position, and the deformity was passively correctable and non-painful. Neurologic investigation was requested, and surgery was indicated for cervical deformity correction.

Passive reduction and cervical-thoracic fixation and arthrodesis were planned from C3 toT3. The head was not included because he had good rotation and flexion-extension range of movement. Surgery was performed in September 24th, 2012. The patient had a good clinical evolution after surgery, and he was discharged at the 5th postoperative day, with a rigid Philadelphia collar that was removed after six weeks. Dysphagia persisted only for solid food, he was able to walk and resumed working as a musician eight weeks after surgery. Electroneuromyography showed sensitive polyneuropathy only, and dysphagia then was attributed to radiotherapy. Ankylosing spondylitis was discarded by the rheumatology team. Neurology consult was requested, and the specialists also ruled out Alzheimer's, Parkinson's, ALS, dystonia and myasthenia. Computed Tomography (CT) and Magnetic Resonance Imaging (MRI) were used for the investigation of spinal or non-spinal metastases, and the exams were all negative.

In the next three months, the patient experienced relief of dysphagia, improvement in walking, he referred no pain, and was capable to look forward. In the sixth-month return consult, however, he complained of worsening of walking posture and thoracolumbar pain. His thoracic kyphosis seemed to be worse at visual examination and lumbar lordosis decreased. Physiotherapy for muscle strengthening was indicated, without any improvement in the next 4 months, when he referred to be worst than ever, complaining again of difficulty swallowing and breathing in the standing position, with relief in the supine position. New X-rays and Computed Tomography (CT) scans were performed. At that time, thoracic kyphosis measured $67 \mathrm{o}$ and lumbar lordosis only 5 degrees (normal thoracic kyphosis should range from 20-50o and lumbar lordosis usually 30-60o). Vertebral bodies looked wedged and some of them were fused anteriorly. Our patient presented high scores of classification of spinal deformity according to Schwab et al. [18] demonstrating severe sagittal deformity and poor health related quality of life.

By October 2013, surgery was offered and planned using a medical imaging software for spinal called Surgimap (available online for free). By this time, deformity could not be passively corrected. Multiple osteotomies were the only way to correct sagittal spinal deformity, and C3 to sacrum arthrodesis was indicated and performed as described below (Figures 1 and 2).

\section{Surgical technique}

In the posterior approach from $\mathrm{C} 3$ to the sacrum, the former instrumentation C3-T3 were tested, and no loosening or breakage were detected. Pedicle screws were inserted by the free-hand technique, except for C7, T1, T6, T10, L2 and L5 were connectors were placed and osteotomies performed. A total of 28 screws were inserted, including the sacropelvic junction in S2. In T6 and T10, Ponte osteotomy and closing wedge pedicle subtraction were made in L2 and laminectomy of L3. After the osteotomies, the position of the screws was checked in fluoroscopy, and titanium rods were folded and fixed to screws 


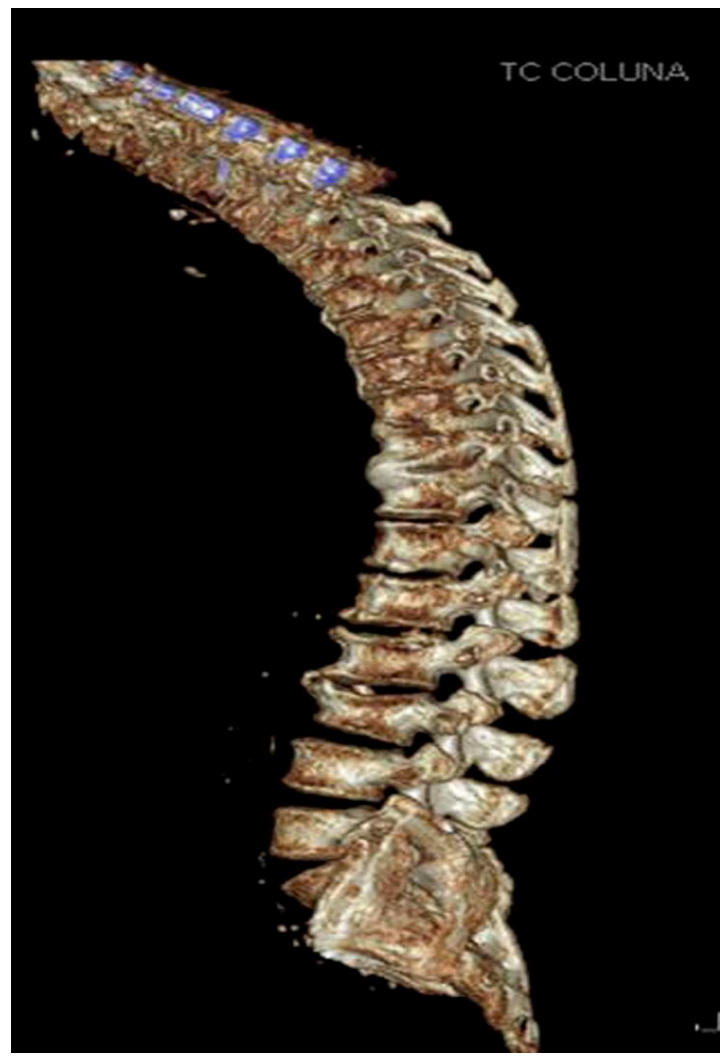

Figure 1: Computed tomography showing the spine deformity preoperatively.

and connected to the cervical instrumentation. A drainage device was installed and the wound was closed. Motor evoked potentials didn't show any abnormality during operation. Two units of blood transfusion were administered during the procedure. Surgery length was 320 minutes.

Patient was admitted in the Intensive Care Unit (ICU) with orotraqueal tube and vasoactive drugs. The tube was removed in the next 24 hours, noradrenaline in approximately 36 hours. After 72 hours, the patient was discharged from the ICU. Physical therapy and ambulation initiated immediately. Patient presented good clinical evolution, and he was discharged from hospital at the 7th postoperative day. Nutrition therapy and therapy with a speech therapist to improve swallowing were initiated, as was physiotherapy. The nasogastric tube was maintained postoperatively and some swallowing improvement happened, but the patient still could not eat normally, and gastrostomy was necessary to improve nutrition. Actinic esophagitis and stenosis were the major causes of dysphagia (Figures 3-5).

In the follow up consult, the patient referred to be satisfied with the procedure and returned to work by the third month. Oncological serum and imaging screening showed no recurrence of the tumor or distant metastasis. After the 13th month consultation, the patient is still happy with results. Some speech therapy is still needed. There was no breakage or instrumentation failure.

\section{Discussion}

Dropped head syndrome and camptocormia associated to highdose radiation therapy is a very rare condition, and mostly associated with Hodgkin's disease. The first case associated with Hodgkin's was described by Johansson et al. [13]. We found in the literature only a single case report of dropped head syndrome induced by chemoradiotherapy, describing a 59-years-old Japanese patient with nasopharyngeal

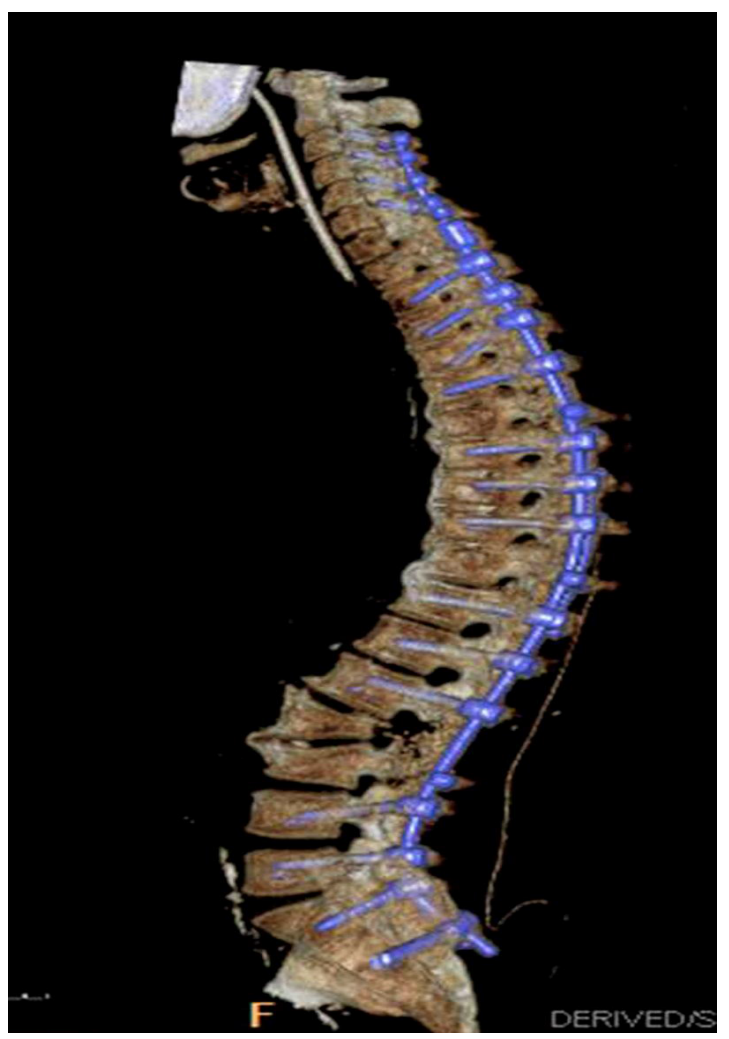

Figure 2: The postoperative correction.

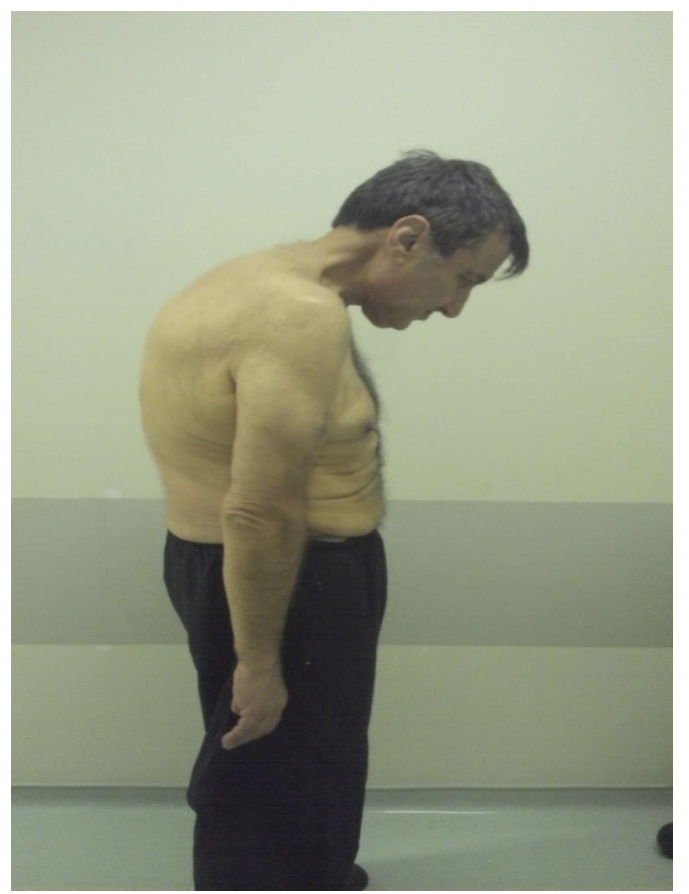

Figure 3: Clinical improvement of patient. Preoperatively. 
Citation: Ono AHA, Zelada FGB, Narazaki DK, Teixeira WGJ, Marcon RM, et al. (2015) Progressive Camptocormia and Dropped Head Syndrome Induced By Chemoradiotherapy for Base of Tongue Carcinoma. Case Report. J Clin Case Rep 5: 613. doi:10.4172/2165-7920.1000613

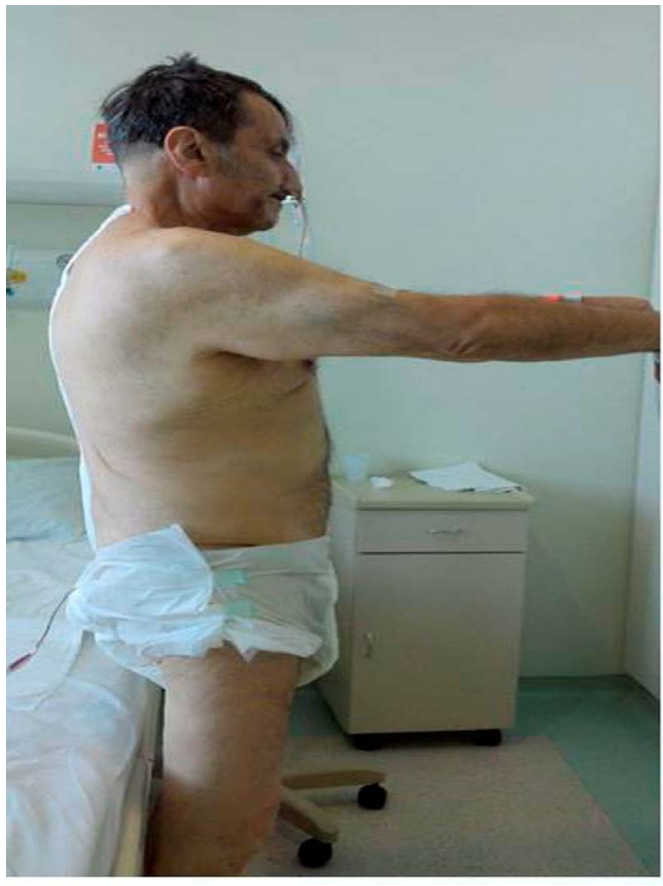

Figure 4: Immediate postoperative period.

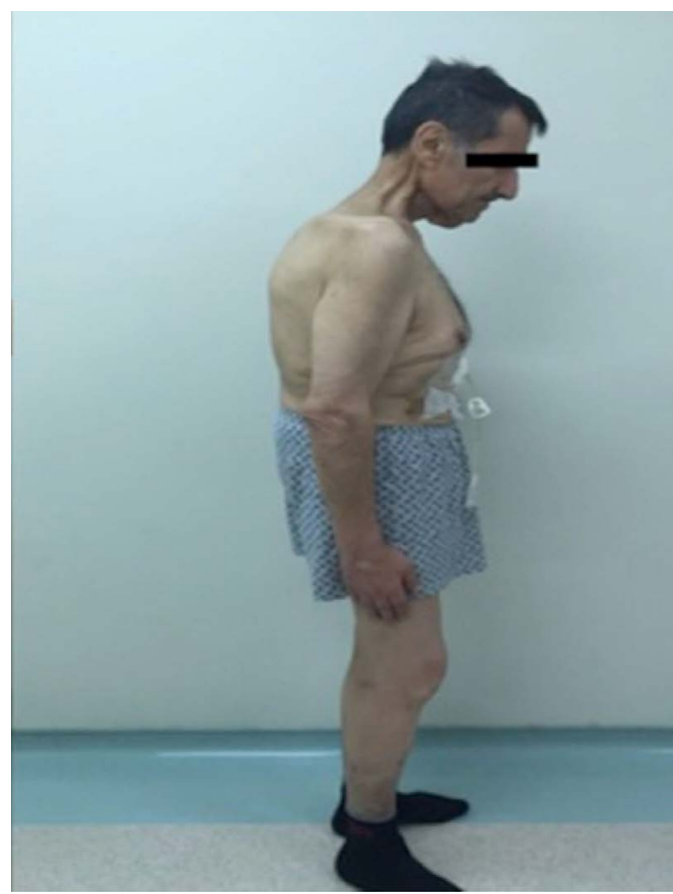

Figure 5: One year follow-up.

carcinoma, described by Hashimoto et al. and treated without surgery. That patient developed symptoms two years after chemoradiotherapy [19]. But the symptoms usually develop later than that, ranging from 10 to more than 20 years [20]. In our patient, symptoms developed in only one year after cancer treatment. The Japanese patient received 39,2 Gy in 22 fractions while ours received 75 Gy in 35 fractions. Additional data is necessary to confirm if the severity and time of symptoms onset are related to radiation doses and fractions and at what proportion.

The physiopathology of dropped head syndrome and camptocormia associated with radiation therapy is still unclear. We believe that high-dose radiation can cause damage to the ventral horn in the upper cervical field and some muscle damages, because in our case the weakness affected progressively the muscle distal to the mantle field (a usual target of radiation therapy). Chemotherapy with radiosensitizing agents combined could enhance severity and the progression of dropped head syndrome, and camptocormia may be due to a synergistic action. This case confirms the hypothesis raised in the Japanese case report that neuromuscular complications can occur due to a combination of high dose radiotherapy and radiosensitizing chemotherapy, and physicians should be aware of this. We would like to emphasize that this condition, independently of the primary tumor or treatment, is extremely debilitating and irreversible, and can lead to poor quality of life and to reduction of performance. It is also life-threatening, because of the progressive dysphagia and dyspnea, which is worrisome especially in patients with large life expectancy, who are free from tumor recurrence or metastasis. We believe that this condition should be treated surgically, and that the rigid collar is only a palliative way to prevent severe deformity in cervical spine, but not capable to correct it. Neglected cases can progress to a severe rigid deformity, which can be difficult to correct surgically.

This is the first surgically treated case of dropped head syndrome and camptocormia induced by radiation therapy for a pharyngeal carcinoma. We believe that surgery is quite challenging in this situation, but it is the only way to improve quality of life in these patients.

\section{References}

1. Finsterer J, Strobl W (2011) Causes of camptocormia. Disabil Rehabil 33 1702-1703.

2. Lenoir T, Guedj N, Boulu P, Guigui P, Benoist M (2010) Camptocormia: the bent spine syndrome, an update. Eur Spine J 19: 1229-1237.

3. Psimaras D, Maisonobe T, Delanian S, Leclercq D, Lenglet T, et al. (2011) Late onset radiation-induced camptocormia. J Neurol 258: 1723-1725.

4. Katz JS, Wolfe GI, Burns DK, Bryan WW, Fleckenstein JL, et al. (1996) Isolated neck extensor myopathy: a common cause of dropped head syndrome. Neurology 46: 917-921.

5. Quinn N (1989) Disproportionate antecollis in multiple system atrophy. Lance 1: 844 .

6. Wadia PM, Tan G, Munhoz RP, Fox SH, Lewis SJ, et al. (2011) Surgical correction of kyphosis in patients with camptocormia due to Parkinson's disease: a retrospective evaluation. J Neurol Neurosurg Psychiatry 82: 364-368.

7. Upadhyaya CD, Starr PA, Mummaneni PV (2010) Spinal deformity and Parkinson disease: a treatment algorithm. Neurosurg Focus 28: E5.

8. Smith JS, Shaffrey Cl, Glassman SD, Berven SH, Schwab FJ, et al. (2011) Risk-benefit assessment of surgery for adult scoliosis: an analysis based on patient age. Spine (Phila Pa 1976) 36: 817-824

9. Sharan AD, Kaye D, Charles Malveaux WM, Riew KD (2012) Dropped head syndrome: etiology and management. J Am Acad Orthop Surg 20: 766-774.

10. Ueda T, Kanda F, Kobessho H, Hamaguchi H, Motomura M (2009) "Dropped head syndrome" caused by Lambert-Eaton myasthenic syndrome. Muscle Nerve 40: 134-136.

11. Suarez GA, Kelly JJ Jr (1992) The dropped head syndrome. Neurology 42 1625-1627.

12. Petheram TG, Hourigan PG, Emran IM, Weatherley CR (2008) Dropped head syndrome: a case series and literature review. Spine (Phila Pa 1976) 33: 47-51.

13. Johansson AS, Erlanson M, Lenner P, Lindh J, Osterman B (1998) [Late sideeffects are common after treatment of Hodgkin's disease. Muscular atrophy following radiotherapy is a neglected risk]. Lakartidningen 95: 44-47. 
Citation: Ono AHA, Zelada FGB, Narazaki DK, Teixeira WGJ, Marcon RM, et al. (2015) Progressive Camptocormia and Dropped Head Syndrome Induced By Chemoradiotherapy for Base of Tongue Carcinoma. Case Report. J Clin Case Rep 5: 613. doi:10.4172/2165-7920.1000613

14. Furby A, Béhin A, Lefaucheur JP, Beauvais K, Marcorelles P, et al. (2010) Lateonset cervicoscapular muscle atrophy and weakness after radiotherapy for Hodgkin disease: a case series. J Neurol Neurosurg Psychiatry 81: 101-104.

15. Ghosh PS, Milone M(2015) Clinical and laboratory findings of 21 patients with radiation-induced myopathy. J Neurol Neurosurg Psychiatry 86: 152-158.

16. Appels C, Goekoop R (2009) Dropped-head syndrome due to high-dose irradiation. J Rheumatol 36: 2316.

17. Rowin J, Cheng G, Lewis SL, Meriggioli MN (2006) Late appearance of dropped head syndrome after radiotherapy for Hodgkin's disease. Muscle Nerve 34: 666-669.
18. Schwab F, Ungar B, Blondel B, Buchowski J, Coe J, et al. (2012) Scoliosis Research Society-Schwab adult spinal deformity classification: a validation study. Spine (Phila Pa 1976) 37: 1077-1082.

19. Hashimoto Y, Maebayashi K, Izumi S, Motegi A, Mitsuhashi N (2012) Dropped head syndrome induced by chemoradiotherapy for nasopharyngeal carcinoma: a case report. Jpn J Clin Oncol 42: 1091-1093.

20. Behin A, Psimaras D, Hoang-Xuan K, Leger JM (2008) Neuropathies in the context of malignancies. Curr Opin Neurol 21: 534-539. 